\title{
Robust Day-Ahead Dispatch for Integrated Power-Heat-Gas Microgrid considering Wind Power Uncertainty
}

\author{
Yang Liu $\mathbb{D}$, Yanli Ye, Xianbang Chen, Huaqiang Li $(\mathbb{D}$, and Yuan Huang $\mathbb{D}$ \\ College of Electrical Engineering, Sichuan University, Chengdu 610065, China \\ Correspondence should be addressed to Yuan Huang; yuanhuang@scu.edu.cn
}

Received 5 May 2019; Revised 1 December 2019; Accepted 7 December 2019; Published 25 February 2020

Academic Editor: Antonio M. Gonçalves de Lima

Copyright (C) 2020 Yang Liu et al. This is an open access article distributed under the Creative Commons Attribution License, which permits unrestricted use, distribution, and reproduction in any medium, provided the original work is properly cited.

\begin{abstract}
Wind power generation has been widely deployed in the modern power system due to the issues of energy crisis and environment pollution. Meanwhile, the microgrid is gradually regarded as a feasible way to connect and accommodate the distributed wind power generations. Recently, more research studies also focus on incorporating various energy systems, for example, heat and gas into the microgrid in terms of satisfying different types of load demands. However, the uncertainty of wind power significantly impacts the economy of the integrated power-heat-gas microgrid. To deal with this issue, this paper presents a two-stage robust model to achieve the optimal day-ahead economic dispatch strategy considering the worst-case wind power scenarios. The first stage makes the initial day-ahead dispatch decision before the observation of uncertain wind power. The additional adjustment action is made in the second stage once the wind power uncertainty is observed. Based on the duality theory and Big-M approach, the original second-stage problem can be dualized and linearized. Therefore, the column-and-constraint generation algorithm can be further implemented to achieve the optimal day-ahead economic dispatch strategy for the integrated power-heat-gas microgrid. The experimental results indicate the effectiveness of the presented approach for achieving operation cost reduction and promoting wind power utilization. The robustness and the economy of the two-stage robust model can be balanced, of which the performances significantly outperform those of the single-stage robust model and the deterministic model.
\end{abstract}

\section{Introduction}

Currently, the generations of the intermittent energy resources (IER), especially the wind power generations, have been widely deployed in the modern power system due to energy crisis and environment pollution $[1,2]$. Meanwhile, the microgrid (MG) has been admitted as a feasible way to connect and accommodate the distributed wind power generations. In research studies [3-5], MG has been proved that it has remarkable ability to reduce the investment cost and the operation cost effectively. Recently, research studies [6-13] have also focused on incorporating various energy forms into MG to compose integrated energy-based $M G$, which is able to satisfy multiple energy demands simultaneously and has great potential to further accommodate IER.

However, there are two natural characteristics of wind challenge of the MG-based distributed wind power generation. The first one is that the uncertainty of wind power may impact the economy of the system operation because of the wind power curtailment [14]. The second one is that the wind power is relatively plentiful at night when the electricity load is relatively low [6]. Therefore, to even out the wind power uncertainty and promote the wind power utilization, research studies claimed that the integration of additional energy equipments and technologies in $M G$ is capable of storing or converting excessive wind power, thus effectively reducing the additional cost of wind power spillage [7-13]. For example, researchers in $[7,8]$ pointed out that the cooperation of the energy storage system (ESS) and the heat storage system (HSS) successfully improves the flexibility of the combined heat and power (CHP) system and the wind power accommodation capability. Nielsen et al. [9] claimed that the deployment of the electric boiler (EB) effectively promotes the wind power utilization and reduces the operation cost by converting wind power to heat energy. Researchers in $[10,11]$ reported that the 
employment of the power-to-gas (PtG) device converting unconsumed wind power to natural gas is highly capable of reducing the wind power spillage in the integrated power and gas system. Recently, studies have concentrated on the integrated power-heat-gas MG $[12,13,15]$. Their experimental results showed great significance with deploying ESS, HSS, PtG, and EB simultaneously in the power-heat-gas MG regarding the effective wind power utilization.

Although novel energy equipments and technologies are helpful for utilizing wind power, it should be realized that the uncertainty of wind power may affect the economic operation of MG due to imprecise prediction of wind [16-19]. Day-ahead ED determines the optimal allocation of the outputs of the controllable generators (CG) [19]. Therefore, day-ahead economic dispatch (ED) $[19,20]$ can be an effective solution to improve the economy of the integrated power-heat-gas MG involving wind power generations [21-24]. Among a wide variety of ED approaches, heuristic algorithms and mathematical optimization approaches have been regarded as the most effective solutions for achieving the optimal ED strategy. For example, researchers in $[25,26]$ employed the differential evolution technique and the real-coded genetic algorithm to study the optimal ED problem for a CHP system, respectively. However, two main disadvantages of the heuristic algorithms cannot be simply neglected $[27,28]$. The first one is that the heuristic algorithms often suffer from local optimal issue. The second one is that the computational time is unstable. Consequently, the mathematical optimization approaches have been widely researched [21-32] due to its advantages of computational efficiency and global optimal solution guarantee.

Li et al. [21] presented a deterministic optimal ED model for a power-gas system. However, in their research, the uncertainty of wind power is not considered, which results in the accuracy loss issue of their optimization. $\mathrm{Li}$ et al. [13] further presented a stochastic optimization (SO) approach to achieve the optimal ED strategy for an integrated power-heat-gas MG involving the wind power uncertainty. However, Li et al. and Bertsimas et al. [22, 23] pointed out that $\mathrm{SO}$ requires explicit and accurate distributions of the uncertainty which is an extreme difficulty in wind prediction. As a result, robust optimization (RO) that needs no accurate distributions of the uncertainty has been incrementally adopted in power system research studies to carry out the optimization tasks [16, 29-32]. Peng et al. [29] employed a single-stage robust optimization model to deal with the uncertainties of wind power and load demand. However, the research reported that the single-stage robust optimization tends to be overconservative. Therefore, the two-stage robust optimization model with "min-max-min" structure has been studied in $[16,30-32]$ to deal with the uncertainty issues. For example, Sun et al. [30] employed a two-stage RO to investigate the unit commitment problem for a system with uncertain wind power. Liu et al. [31] introduced a twostage RO to achieve cooptimization of energy and reserve dispatch for an integrated power and gas system. Peng and Liu [16] presented a two-stage robust hydrothermal-wind
ED model to improve the flexibility of the power system operation. According to the results of above researches although $\mathrm{RO}$ has successfully solved unit commitment [30], cooptimization of energy and reserve [31], energy management [32], and day-ahead ED problems [16], few research studies have investigated the potential of $\mathrm{RO}$ in enabling optimal day-ahead ED for the integrated powerheat-gas MG.

Motivated by the aforementioned research studies, this paper employs $\mathrm{RO}$ as the underlying approach to achieve the optimal day-ahead ED strategy for the integrated powerheat-gas MG involving the uncertain wind power. Firstly, a two-stage ED model is presented. In the first stage, the initial day-ahead decision is made before the realization of the wind power uncertainty. Once the uncertainty is observed, the additional corrective action is decided in the second stage to compensate the power imbalance. Secondly, regarding the nature characteristics of the wind power, an uncertainty set is constructed to describe the uncertainty of wind power. Thirdly, the strong duality theory and Big-M theory are employed to transform the original two-stage model into a mixed-integer linear problem (MILP). As a result, the original model is able to be solved directly by using the column-and-constraint generation (C\&CG) algorithm.

The rest of the paper is organized as follows: Section 2 constructs the two-stage model for the integrated powerheat-gas MG involving the uncertain wind power; Section 3 constructs the uncertainty set of wind power and presents the model solution; Section 4 shows and discusses the experiment results; Section 5 concludes the paper.

\section{Two-Stage Day-Ahead ED for the Integrated Power-Heat-Gas MG}

2.1. Objective Function. This paper focuses on achieving the optimal day-ahead ED strategy for integrated power-heatgas MG involving the uncertain wind power. Firstly, the structure of the researched MG is illustrated in Figure 1.

The two-stage robust model can be presented as the following equation:

$$
\begin{array}{ll}
\min _{x} & \left\{C_{\mathrm{da}}(x)+\max _{u} \min _{y} C_{\mathrm{da}}^{+}(x, u, y)\right\} \\
\text { s.t. } & A_{\mathrm{da}}(x)=0, A_{\mathrm{da}}^{+}(y)=0 .
\end{array}
$$

In equation (1), $x$ represents the first-stage dispatch variables. The details of $x$ are listed in Table $1 . C_{\mathrm{da}}$ is the dayahead operation cost of MG, which is made before the observation of the uncertain wind power. The details of $C_{\mathrm{da}}$ are shown in the following equation:

$$
\begin{aligned}
C_{\mathrm{da}}= & \sum_{t=1}^{T}\left(\lambda_{\mathrm{gas}}(t) G_{\mathrm{gas}}(t)+a_{\mathrm{FC}} \cdot P_{\mathrm{FC}}(t)+b_{\mathrm{FC}} \cdot S_{\mathrm{FC}}(t)\right. \\
& -\lambda_{\text {Grid }}^{\text {sell }}(t) P_{\mathrm{Grid}}^{\text {sold }}(t)+\lambda_{\text {Grid }}^{\text {buy }}(t) P_{\text {Grid }}^{\text {purchased }}(t)+\lambda_{\text {wind }}(t) \\
& \left.\cdot\left(P_{\text {forecasted }}(t)-P_{\text {firstwind }}(t)\right)\right),
\end{aligned}
$$




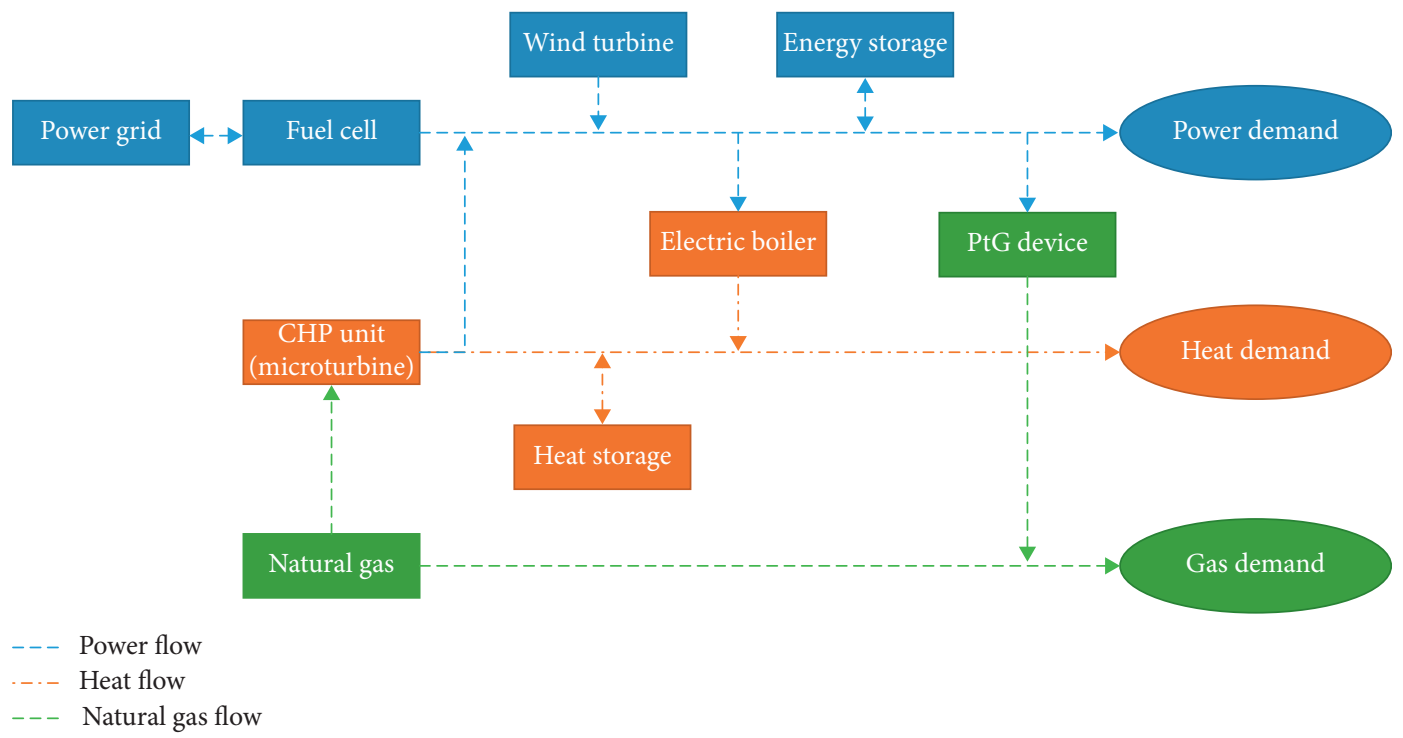

Figure 1: The structure of the power-heat-gas MG.

TABLE 1: Details of the day-ahead dispatch scheduling variables.

\begin{tabular}{lc}
\hline Name & Abbreviation \\
\hline Output of the fuel cell & $P_{\mathrm{FC}}$ \\
Consumption of gas supply & $G_{\text {gas }}$ \\
Sold power with the power grid & $P_{\text {grid }}^{\text {sold }}$ \\
Purchased power with the power grid & $P_{\text {grid }}^{\text {purchased }}$ \\
Wind power & $P_{\text {firstwind }}$ \\
\hline
\end{tabular}

where $a_{\mathrm{FC}}$ and $b_{\mathrm{FC}}$ are the coefficients of the fuel cell (FC); $S_{\mathrm{FC}}$ represents the ON/OFF status of FC; $\lambda_{\text {gas }}$ is the price of the natural gas; $\lambda_{\text {Grid }}^{\text {buy }}$ and $\lambda_{\text {Grid }}^{\text {sell }}$ are the prices of the sold and purchased power with the grid; $P_{\text {Grid }}^{\text {sold }}$ and $P_{\text {Grid }}^{\text {purchased }}$ represent the sold and purchased power; and $P_{\text {firstwind }}$ and $P_{\text {forecasted }}$ represent the scheduled wind power in the first stage and the forecasted wind power, respectively.

$C_{\mathrm{da}}$ consists of the operation cost of FC, the cost of the natural gas consumption, the benefit of the interaction with the grid, and the penalty cost of the wind power curtailment [33].

The first-stage strategy only considers the forecasted value of the wind power. However, the imprecise prediction may lead to power imbalance in the power-heat-gas MG. Therefore, in the second stage, the decision $C_{\mathrm{da}}^{+}$is the adjustment action after the observation of the wind power uncertainty.

Additionally, in equation (1), the second-stage max function searches for the worst-case scenarios which represents that the wind power is the most insufficient. The inner layer min function minimizes the adjustment cost under the discovered worst-case scenarios. $u$ stands for the wind power. $y$ is the second-stage adjustment variables. The details of $y$ are listed in Table 2. $C_{\mathrm{da}}^{+}$is shown in the following equation:
TABLE 2: Details of the adjustment scheduling variables.

\begin{tabular}{lc}
\hline Name & Abbreviation \\
\hline Up/downregulation of CG & $P_{\mathrm{CG}}^{\text {up }}, P_{\mathrm{CG}}^{\text {down }}$ \\
Up/downregulation of natural gas & $G_{\mathrm{CG}}^{\mathrm{u}}, G_{\mathrm{CG}}^{\text {down }}$ \\
Adjustment of sold power & $P_{\text {Baldanced }}^{\text {sald }}$ \\
Adjustment of purchased power & $P_{\text {Balanced }}^{\text {purchased }}$ \\
Actual wind power & $P_{\text {secondwind }}$ \\
\hline
\end{tabular}

$$
\begin{aligned}
C_{\mathrm{da}}^{+}= & \sum_{t=1}^{T}\left(\lambda_{C G}^{\mathrm{up}} \cdot P_{C G}^{\text {up }}(t)+\lambda_{C G}^{\text {down }} \cdot P_{C G}^{\text {down }}(t)+\lambda_{\text {gas }}^{\text {up }} \cdot G_{\text {gas }}^{\text {up }}(t)\right. \\
& +\lambda_{\text {gas }}^{\text {down }} \cdot G_{\text {gas }}^{\text {down }}(t)+\lambda_{\text {Balance }}^{\text {sold }}(t) P_{\text {Balance }}^{\text {sold }}(t) \\
& -\lambda_{\text {Balance }}^{\text {purchased }}(t) P_{\text {Balance }}^{\text {purchased }}(t)+\lambda_{\text {wind }}(t) \\
& \left.\cdot\left(P_{\text {wind }}^{s}(t)-P_{\text {secondwind }}(t)\right)\right),
\end{aligned}
$$

where and $\lambda_{\mathrm{CG}}^{\text {down }}$ are the prices of the up/downregulation of CG; $\lambda_{\text {gas }}^{\text {up }}$ and $\lambda_{\text {gas }}^{\text {down }}$ are the prices of the up/downregulation of the natural gas supply; and $P_{\text {wind }}^{s}$ is the uncertain wind power.

$\mathrm{C}_{\mathrm{da}}^{+}$consists of the additional adjustment cost of CG, the additional adjustment cost of natural gas supply, the adjustment cost of the exchange power with the grid, and the penalty cost of the wind curtailment once the uncertain wind is observed. In this paper, CG represents FC, microturbine, $\mathrm{EB}$, and $\mathrm{PtG}$.

$A_{\mathrm{da}}(\cdot)$ and $A_{\mathrm{da}}^{+}(\cdot)$ in equation (1) represent the firststage constraints and adjustment constraints, respectively. The details of these constraints are discussed in Section 2.2 . 


\subsection{Constraints}

\subsubsection{First-Stage Constraints}

(1) Energy Balance Constraints. The energy balances for various types of energies in the integrated power-heat-gas MG are guaranteed as follows:

$$
\begin{aligned}
P_{\mathrm{FC}}(t)+P_{\mathrm{MT}}(t)+P_{\mathrm{ESS}}^{\mathrm{dis}}(t)+P_{\text {firstwind }}(t)+P_{\mathrm{Grid}}^{\text {purchased }}(t) & =P_{\mathrm{PtG}}(t)+P_{\text {load }}(t)+P_{\mathrm{EB}}(t)+P_{\mathrm{Grid}}^{\text {sold }}(t)+P_{\mathrm{ESS}}^{\mathrm{ch}}(t), \\
Q_{\mathrm{MT}}(t)+Q_{\mathrm{HSS}}^{\mathrm{dis}}(t)+Q_{\mathrm{EB}}(t) & =Q_{\text {load }}(t)+Q_{\mathrm{HSS}}^{\mathrm{ch}}(t) \\
G_{\mathrm{gas}}(t)+G_{\mathrm{PtG}}(t) & =G_{\text {load }}(t)+G_{\mathrm{MT}}(t)
\end{aligned}
$$

where $P_{\mathrm{ESS}}^{\mathrm{dis}}$ and $P_{\mathrm{ESS}}^{\mathrm{ch}}$ are the discharging/charging power of ESS; $P_{\mathrm{MT}}$ is the power output of the microturbine; $P_{\mathrm{PtG}}$ and $P_{\mathrm{EB}}$ are the electric power consumed by PtG and EB; $Q_{\mathrm{HSS}}^{\text {dis }}$ and $Q_{H S S}^{\text {ch }}$ are the discharging/charging power of HSS; $P_{\text {load }}, Q_{\text {load }}$, and $G_{\text {load }}$ are the electric, heat, and gas load demands, respectively; $Q_{\mathrm{MT}}$ and $Q_{\mathrm{EB}}$ are the heat energy generated by the microturbine and $\mathrm{EB}$; $G_{\text {gas }}$ is the total amount of supplied natural gas; $G_{\mathrm{MT}}$ is the gas consumption of the microturbine; and $G_{\mathrm{PtG}}$ is the gas generated by PtG.

In our model, the microturbine is a back-pressure CHP which generates electric power by consuming natural gas. Its converting ratio of the electric and heat power output is a constant value [34]. Therefore, $Q_{\mathrm{MT}}, P_{\mathrm{MT}}$, and $G_{\mathrm{MT}}$ can be presented in the following equation:

$$
\begin{aligned}
& Q_{\mathrm{MT}}(t)=\eta_{\mathrm{MT}} \cdot P_{\mathrm{MT}}(t), \\
& P_{\mathrm{MT}}(t)=\eta_{\text {gas }} \cdot G_{\mathrm{MT}}(t),
\end{aligned}
$$

where $\eta_{\mathrm{MT}}$ is the constant ratio of converting the electric and heat power output; $\eta_{\mathrm{MT}}$ is the converting efficiency of the gas consumption and the electricity output; similarly, $G_{\mathrm{PtG}}$ and $Q_{\mathrm{EB}}$ can also be calculated as follows:

$$
\begin{aligned}
G_{\mathrm{PtG}}(t) & =\eta_{\mathrm{PtG}} \cdot P_{\mathrm{PtG}}(t), \\
Q_{\mathrm{EB}}(t) & =\eta_{\mathrm{EB}} \cdot P_{\mathrm{EB}}(t),
\end{aligned}
$$

where $\eta_{\mathrm{PtG}}$ and $\eta_{\mathrm{EB}}$ represent the conversion efficiency of $\mathrm{PtG}$ and $\mathrm{EB}$. In this paper, $\eta_{\mathrm{MT}}, \eta_{\mathrm{gas}}, \eta_{\mathrm{PtG}}$, and $\eta_{\mathrm{EB}}$ are equal to $0.9,0.85,0.85$, and 0.8 , respectively $[12,34-37]$.

(2) CG Constraints. The constraints of CG include ramping rate and the limit of power output, as shown as follows:

$$
\begin{gathered}
0 \leq P_{\mathrm{CG}, i}(t) \leq S_{\mathrm{CG}, i}(t) \cdot P_{\mathrm{CG}, i}^{\max }, \\
-R_{\mathrm{CG}, i} \leq P_{\mathrm{CG}, i}(t)-P_{\mathrm{CG}, i}(t-1) \leq R_{\mathrm{CG}, i},
\end{gathered}
$$

where $P_{\mathrm{CG}, i}^{\max }$ represents the maximum power output of the $i^{\text {th }}$ $\mathrm{CG} ; R_{\mathrm{CG}, i}^{\mathrm{up}}$ is the maximum ramping rate of the $i^{\text {th }} \mathrm{CG}$; and $S_{\mathrm{CG}, i}$ represents the ON/OFF status of CG.

(3) Storage System Constraints. The storage system (SS) mainly includes ESS and HSS. In each hour $t$, the energy balance of SS can be constrained as follows:

$$
\begin{aligned}
E_{\mathrm{SS}, i}(t) & =E_{\mathrm{SS}, i}(t-1)+P_{\mathrm{SS}, i}^{\mathrm{ch}}(t) \eta_{\mathrm{SS}, i}^{\mathrm{ch}}-\frac{P_{\mathrm{SS}, i}^{\mathrm{dis}}(t)}{\eta_{\mathrm{SS}, i}^{\mathrm{dis}}}, \\
0 & \leq P_{\mathrm{SS}, i}^{\mathrm{ch}}(t) \leq P_{\mathrm{SS}, i}^{\mathrm{ch}, \max } S_{\mathrm{SS}, i}^{\mathrm{ch}}(t), \\
0 & \leq P_{\mathrm{SS}, i}^{\mathrm{dis}}(t) \leq P_{\mathrm{SS}, i}^{\mathrm{dis}, \max } \cdot\left(1-S_{\mathrm{SS}, i}^{\mathrm{ch}}(t)\right),
\end{aligned}
$$

where $E_{\mathrm{SS}, i}$ is the stored energy of the $i^{\text {th }} \mathrm{SS} ; P_{\mathrm{SS}, i}^{\mathrm{dis}}$ and $P_{\mathrm{SS}, i}^{\mathrm{ch}}$ are the discharging/charging power of the $i^{\text {th }} \mathrm{SS} ; \eta_{\mathrm{SS}, i}^{\mathrm{ch}}$ and $\eta_{\mathrm{SS}, i}^{\mathrm{dis}}$ are the charging and discharging rates; $P_{\mathrm{SS}, i}^{\mathrm{dis}, \max }$ and $P_{\mathrm{SS}, i}^{\mathrm{ch}, i}$ are the upper bounds of the allowable discharging/discharging power; and $S_{\mathrm{SS}, i}^{\mathrm{ch}}$ is a binary variable, which represents the ON/OFF status of charging mode.

Moreover, the final stored energy should be equal to its initial stored energy, as shown in the following equation:

$$
E_{\mathrm{SS}, i}(T)=E_{\mathrm{SS}, i}(0)
$$

(4) Interaction Constraints. The exchange power between MG and the power grid should be limited as follows:

$$
\begin{aligned}
& 0 \leq P_{\text {Grid }}^{\text {purchased }}(t) \leq P_{\text {Grid }}^{\max } \cdot S_{\text {Grid }}^{\text {purchased }}(t), \\
& 0 \leq P_{\text {Grid }}^{\text {sold }}(t) \leq P_{\text {Grid }}^{\max } \cdot\left(1-S_{\text {Grid }}^{\text {purchased }}(t)\right),
\end{aligned}
$$

where $P_{\mathrm{Grid}}^{\max }$ is the maximum exchange power with the grid and $S_{\text {Grid }}^{\text {purchased }}$ is a binary variable, which represents the ON/ OFF status of the purchasing mode.

(5) Wind Power Constraint. The scheduled wind power in the first stage should not exceed the forecasted wind power [30], as shown in the following equation:

$$
0 \leq P_{\text {firstwind }}(t) \leq P_{\text {forecasted }}(t) \text {. }
$$

\subsubsection{Adjustment Constraints}

(1) Energy Balance Constraints. Once the uncertain wind power is observed, the second-stage decision should be made to compensate the power imbalance in MG. Power, heat, and gas energy balances should be satisfied after the adjustment action in the second stage as shown in the following equation: 


$$
\begin{aligned}
& P_{\mathrm{FC}}(t)+P_{\mathrm{FC}}^{\mathrm{up}}(t)-P_{\mathrm{FC}}^{\mathrm{down}}(t)+P_{\mathrm{MT}}(t)+P_{\mathrm{MT}}^{\mathrm{up}}(t)-P_{\mathrm{MT}}^{\mathrm{down}}(t)+P_{\mathrm{ESS}}^{\text {dis,adjust }}(t)+P_{\text {secondwind }}(t)+P_{\mathrm{Grid}}^{\text {purchased }}(t)+P_{\mathrm{Balance}}^{\text {purchased }}(t) \\
&=P_{\mathrm{PtG}}(t)+P_{\mathrm{PtG}}^{\mathrm{up}}(t)-P_{\mathrm{PtG}}^{\text {down }}(t)+P_{\text {load }}(t)+P_{\mathrm{EB}}(t)+P_{\mathrm{EB}}^{\text {up }}(t)-P_{\mathrm{EB}}^{\text {down }}(t)+P_{\mathrm{Grid}}^{\text {sold }}(t)+P_{\text {Balance }}^{\text {sold }}(t)+P_{\mathrm{ESS}, i}^{\text {ch,adjust }}(t), \\
& Q_{\mathrm{MT}}(t)+Q_{\mathrm{MT}}^{\mathrm{up}}(t)-Q_{\mathrm{MT}}^{\text {down }}(t)+Q_{\mathrm{HSS}}^{\text {dis,adjust }}(t)+Q_{\mathrm{EB}}(t)+Q_{\mathrm{EB}}^{\text {up }}(t)-Q_{\mathrm{EB}}^{\text {down }}(t)=Q_{\text {load }}(t)+Q_{\mathrm{HSS}}^{\text {ch,adjust }}(t), \\
& G_{\text {supply }}(t)+G_{\text {supply }}^{\text {up }}(t)-G_{\text {supply }}^{\text {down }}(t)+G_{\mathrm{PtG}}(t)+G_{\mathrm{PtG}}^{\mathrm{up}}(t)-G_{\mathrm{PtG}}^{\text {down }}(t) \\
&= G_{\mathrm{MT}}(t)+G_{\mathrm{MT}}^{\mathrm{up}}(t)-G_{\mathrm{MT}}^{\text {down }}(t)+G_{\text {load }}(t),
\end{aligned}
$$

where $P_{\mathrm{MT}}^{\mathrm{up}}, P_{\mathrm{FC}}^{\mathrm{up}}, P_{\mathrm{PtG}}^{\mathrm{up}}, P_{\mathrm{EB}}^{\mathrm{up}}, P_{\mathrm{MT}}^{\text {down }}, P_{\mathrm{FC}}^{\text {down }}, P_{\mathrm{PtG}}^{\text {down }}$, and $P_{\mathrm{EB}}^{\text {down }}$ are the up/downregulation power output of the microturbine, FC, $\mathrm{PtG}$, and $\mathrm{EB} ; Q_{\mathrm{MT}}^{\mathrm{up}}, Q_{\mathrm{EB}}^{\text {up }}, Q_{\mathrm{MT}}^{\text {down }}$, and $Q_{\mathrm{EB}}^{\text {down }}$ are the up/downregulation heat output of the microturbine and EB; $G_{\mathrm{gas}}^{\mathrm{up}}, G_{\mathrm{PtG}}^{\mathrm{up}}, G_{\mathrm{gas}}^{\text {down }}$, and $Q_{\mathrm{PtG}}^{\text {down }}$ are the up/downregulation gas output of the gas supplier and PtG; $P_{\mathrm{ESS}}^{\text {ch,adjust }}, P_{\mathrm{ESS}}^{\text {dis,adjust }}$, $Q_{\mathrm{HSS}}^{\text {ch,adjust }}$, and $Q_{\mathrm{HSS}}^{\text {diss,ast }}$ are the charging and discharging power of ESS and HSS after the adjustment.

\section{(2) Regulation Constraints}

$$
\begin{gathered}
S_{\mathrm{CG}, i}(t) \cdot 0 \leq P_{\mathrm{CG}, i}(t)+P_{\mathrm{CG}, i}^{\mathrm{up}}(t)-P_{\mathrm{CG}, i}^{\mathrm{down}}(t) \leq S_{\mathrm{CG}, i}(t) \cdot P_{\mathrm{CG}, i}^{\mathrm{max}}, \\
-R_{\mathrm{CG}, i} \leq P_{\mathrm{CG}, i}(t)+P_{\mathrm{CG}, i}^{\mathrm{up}}(t)-P_{\mathrm{CG}, i}^{\mathrm{down}}(t)-\left(P_{\mathrm{CG}, i}^{\mathrm{up}}(t-1)\right. \\
\left.+P_{\mathrm{CG}, i}^{\mathrm{up}}(t-1)-P_{\mathrm{CG}, i}^{\mathrm{down}}(t-1)\right) \leq R_{\mathrm{CG}, i},
\end{gathered}
$$

$$
\begin{aligned}
& 0 \leq P_{\mathrm{CG}, i}^{\mathrm{up}}(t) \leq S_{\mathrm{CG}, i}^{\mathrm{up}}(t) \cdot P_{\mathrm{CG}}^{\mathrm{max}}, \\
& 0 \leq P_{\mathrm{CG}, i}^{\mathrm{down}}(t) \leq\left(1-S_{\mathrm{CG}, i}^{\mathrm{up}}(t)\right) \cdot P_{\mathrm{CG}, i}^{\max },
\end{aligned}
$$

where $S_{\mathrm{CG}, i}^{\mathrm{up}}$ is a binary variable, which represents the $\mathrm{ON} /$ OFF status of the $i^{\text {th }}$ CG. Equations (15)-(17) ensure that the ramping rate and the power output are within the permissible range.

Similarly, the gas power regulation of the gas supplier and the regulation of SS should be within the allowable range. And simultaneous up- and downregulation of the gas supplier and SS should be avoided, which is expressed as follows:

$$
\begin{aligned}
0 & \leq G_{\text {supply }}^{\text {up }}(t) \leq S_{\text {supply }}^{\text {up }}(t) \cdot G_{\text {supply }}^{\text {max }}, \\
0 & \leq G_{\text {supply }}^{\text {down }}(t) \leq\left(1-S_{\text {supply }}^{\text {up }}(t)\right) \cdot G_{\text {supply }}^{\text {max }} \\
E_{\mathrm{SS}, i}^{\text {adjust }}(t) & =E_{\mathrm{SS}, i}^{\text {adjust }}(t-1)+P_{\mathrm{SS}, i}^{\text {ch,adjust }}(t) \cdot \eta_{\mathrm{SS}, i}^{\text {ch }}-\frac{P_{\mathrm{SS}, i}^{\text {dis,adjust }}(t)}{\eta_{\mathrm{SS}, i}^{\text {dis }}}, \\
0 & \leq P_{\mathrm{S} S, i}^{\text {ch,adjust }}(t) \leq P_{\mathrm{SS}, i}^{\mathrm{ch}, \max } \cdot S_{\mathrm{SS}, i}^{\mathrm{ch}}(t), \\
0 & \leq P_{\mathrm{SS}, i}^{\mathrm{dis,adjust}}(t) \leq P_{\mathrm{SS}, i}^{\mathrm{dis}, \mathrm{max}} \cdot\left(1-S_{\mathrm{SS}, i}^{\mathrm{ch}}(t)\right),
\end{aligned}
$$

where $P_{\mathrm{SS}, i}^{\text {ch,adjust }}$ and $P_{\mathrm{SS}}^{\text {dis,adjust }}$ are the charging and discharging power of the $i^{\text {th }} S S$ after the adjustment and $E_{\mathrm{SS}, i}^{\text {adjust }}$ is the stored energy after the adjustment. Notably, charging/ discharging mode remains the same status as in the firststage decision.

\section{(3) Interaction Constraints}

$$
\begin{aligned}
& 0 \leq P_{\text {Balance }}^{\text {sold }}(t) \leq\left(1-S_{\text {Balance }}^{\text {purchased }}(t)\right) \cdot P_{\text {Balance }}^{\max }(t), \\
& 0 \leq P_{\text {Balance }}^{\text {purchased }}(t) \leq S_{\text {Balance }}^{\text {purchased }}(t) \cdot P_{\text {Balance }}^{\max }(t), \\
& 0 \leq P_{\text {Grid }}^{\text {sold }}(t)+P_{\text {Balance }}^{\text {sold }}(t) \leq P_{\mathrm{Grid}}^{\max } \cdot\left(1-S_{\text {Grid }}^{\text {purchased }}(t)\right), \\
& 0 \leq P_{\text {Grid }}^{\text {purchased }}(t)+P_{\text {Balance }}^{\text {purchase }}(t) \leq P_{\text {Grid }}^{\text {max }} \cdot S_{\text {Grid }}^{\text {purched }}(t),
\end{aligned}
$$

where $S_{\text {Balance }}^{\text {purchase }}$ is a binary variable, which represents the selling/purchasing power status; $P_{\text {Balance }}^{\max }$ is the maximum adjustment power with the grid; the limits of the sold and purchased adjustment power should be constrained by equation (21). After the adjustment, the selling/purchasing mode with the grid keeps the same as the first-stage exchange status. The exchange power with the grid should not exceed the permissible range, which is expressed in equation (22).

(4) Wind Power Constraint. After the uncertainty of the wind power is revealed in the second stage, the actual wind power should be less than the observed wind power, which is indicated as follows:

$$
0 \leq P_{\text {secondwind }}(t) \leq P_{\text {wind }}^{s}(t) .
$$

\section{Uncertainty Modeling and Model Solution}

3.1. Uncertainty Set. To guarantee the operation robustness, the formulation protects against all possible outputs of the wind power within an uncertainty set $U$. The budget of uncertainty $\Gamma$ is also employed to adjust the degree of the conservativeness during the dispatch period $T$. The entire uncertainty set $U$ adopted by this paper is presented in the following equation:

$$
U=\left\{\begin{array}{c}
P_{\text {wind }}^{s}: P_{\text {forecasted }}(t)-P_{\text {wind }}^{\text {error }}(t) \leq P_{\text {wind }}^{s}(t) \leq P \\
\sum_{t=1}^{T}\left|\frac{P_{\text {wind }}^{s}(t)-P_{\text {forecasted }}(t)}{P_{\text {forecasted }}(t)}\right| \leq \Gamma
\end{array}\right\},
$$

where $P_{\text {wind }}^{\text {error }}$ is the maximum forecasted error of the wind power. The value of $\Gamma$ controls the conservativeness of the optimized result. 
3.2. Compact Formulation. In terms of facilitating the presentation, the aforementioned objective function equations (1)-(3) and the constraints of the two-stage ED model equations (4)-(24) can be rewritten into a compact form:

$$
\begin{cases}\min _{x} & \mathbf{a}^{T} \mathbf{x}+\max _{u \in U} \min _{y \in(x, u)} \mathbf{b}^{T} \mathbf{y} \\ \text { s.t. } & A x \leq d \\ & (\mathbf{x}, \mathbf{u})=\left\{\mathbf{y}: \mathbf{B} \mathbf{x}+\mathbf{C y} \leq \mathbf{e}, \mathbf{D}_{u} \mathbf{y} \leq \mathbf{u}\right\},\end{cases}
$$

where $\mathbf{a}, \mathbf{b}, \mathbf{A}, \mathbf{B}, \mathbf{C}$, and $\mathbf{D}_{\mathbf{u}}$ are the constant matrices and $\mathbf{d}$ and e represent vectors.

3.3. Model Decomposition. The presented model has a complicated min-max-min structure, which cannot be solved directly. However, C\&CG has been proved to be an effective algorithm for solving the complex two-stage model with remarkable computational efficiency and convergence capability [38]. Therefore, C\&CG is employed to solve the presented two-stage ED model. Based on C\&CG, the original model is firstly decomposed into the master problem (MP) and the subproblem (SP). The optimal first-stage decision $\mathbf{x}$ is decided in MP given the possible scenarios from SP, while SP searches for the possible worst-case scenarios given the first-stage decisions from MP. Afterwards, the problem is able to converge to the optimal solution during the iterations between MP and SP.

Therefore, MP can be rewritten as follows:

$$
\begin{array}{cc}
\min _{x} & c^{T} x+\eta \\
\text { s.t. } & \mathbf{A} x \leq \mathbf{d}, \\
\eta \geq \mathbf{b}^{T} \mathbf{y}^{k}, \\
\mathbf{B} \mathbf{x}+\mathbf{C y}^{k} \leq \mathbf{e}, \\
\mathbf{D}_{u} \mathbf{y}^{k} \leq \mathbf{u}_{k}^{*},
\end{array}
$$

where $\eta$ is an auxiliary variable and $\mathbf{y}^{k}$ and $\mathbf{u}^{k}$ are the optimal adjustment decisions and the possible wind power scenarios at the $k^{\text {th }}$ iteration, respectively. By gradually identifying the possible wind power scenarios at each iteration, the updated constraint equations (27)-(29) are added into MP.Given the first-stage decision $\mathbf{x}^{*}$ of MP, SP can be presented as

$$
\begin{array}{ll}
\max _{\mathbf{u} \in U} \min _{y \in(\mathbf{x} * \mathbf{u})} & \mathbf{b}^{T} \mathbf{y} \\
\text { s.t. } & \left(\mathbf{x}^{*}, \mathbf{u}\right)=\left\{\mathbf{y}: \mathbf{B} \mathbf{x}^{*}+\mathbf{C y} \leq \mathbf{e}, \mathbf{D}_{u} \mathbf{y} \leq \mathbf{u}\right\} .
\end{array}
$$

3.4. Transformation of SP. SP contains a max-min structure, which is difficult to be solved. Therefore, strong duality theory is applied to transform SP into an equivalent singlestage problem SP1 as shown in the following equation:

$$
\begin{array}{ll}
\max _{\mathbf{u}, \boldsymbol{\pi}_{1}, \boldsymbol{\pi}_{2}} & \mathbf{e}^{\mathrm{T}} \boldsymbol{\pi}_{1}-\mathbf{x}^{* \mathrm{~T}} \mathbf{B}^{\mathrm{T}} \boldsymbol{\pi}_{1}+\mathbf{u}^{T} \boldsymbol{\pi}_{2} \\
\text { s.t. } & \mathbf{C}^{T} \boldsymbol{\pi}_{1}+\mathbf{D}_{u}^{T} \boldsymbol{\pi}_{2}=\mathbf{b}, \boldsymbol{\pi}_{1} \leq 0,
\end{array}
$$

where $\pi_{1}$ and $\pi_{2}$ are the dual variables for the constraints of SP equation (30) and $\mathbf{u}^{\mathrm{T}} \boldsymbol{\pi}_{2}$ is a nonconvex bilinear term, which also leads to the difficulties of directly solving $\mathrm{SP} 1$. However, $\mathbf{u}$ only contains the forecasted value $\mathbf{u}^{0}$, the upper limit value $\mathbf{u}^{+}$, or the lower limit value $\mathbf{u}^{-}$, which is not continuous. Therefore, $\mathbf{u}^{\mathrm{T}} \boldsymbol{\pi}_{2}$ can be linearized by applying the Big-M method. Meanwhile, the uncertain budget is also introduced to adjust the uncertainty set. As a result, the transformed SP2 can be expressed by equation (32) which is able to be solved in a feasible way:

$$
\begin{array}{cl}
\max _{u, \pi_{1}, \pi_{2}} & \mathbf{e}^{\mathrm{T}} \pi_{1}-\mathbf{x}^{*^{\mathrm{T}}} \mathbf{B}^{\mathrm{T}} \pi_{1}+\mathbf{u}^{+} \pi_{2}^{+}+\mathbf{u}^{-} \pi_{2}^{-}+\mathbf{u}^{0}\left(1-\pi_{2}^{+}-\pi_{2}^{-}\right), \\
\text {s.t. } & \mathbf{C}^{T} \pi_{1}+\mathbf{D}_{u}^{T} \pi_{2}=\mathbf{b}, \pi_{1} \leq 0-M\left(1-\mu_{t}^{u}\right)+\pi_{2, t} \\
& \leq \pi_{2, t}^{+} \leq M\left(1-\mu_{t}^{u}\right)+\pi_{2, t}-M\left(1-\mu_{t}^{l}\right)+\pi_{2, t} \leq \pi_{2, t}^{-} \\
& \leq M\left(1-\mu_{t}^{l}\right)+\pi_{2, t}-M \mu_{t}^{u} \leq \pi^{+} \leq M \mu_{t}^{u},-M \mu_{t}^{l} \leq \pi_{2, t}^{-} \\
& \leq M \mu_{t}^{l} \mu_{t}^{u}+\mu_{t}^{l} \leq 1, \sum_{t}\left(\mu_{t}^{u}+\mu_{t}^{l}\right) \leq \Gamma,
\end{array}
$$

where $\pi_{2}^{+}$and $\pi_{2}^{-}$represent the positive and negative values of $\pi_{2}$, corresponding to the upper and lower bounds of the wind power and $M$ is a large enough constant.

3.5. Model Solution Using C\&CG. The solution of the model using C\&CG includes the following major steps given in Table 3.

\section{Experimental Result}

In this section, a number of evaluations are carried out to demonstrate the effectiveness of the presented ED optimization approach for an integrated power-heat-gas MG. The parameters of FC, the microturbine, EB, and the PtG devices are listed in Table 4. The parameters of ESS and HSS are listed in Table 5. The cost of gas and the adjustment cost of gas are $0.85 \$ / \mathrm{m}^{3}$ and $1.05 \$ / \mathrm{m}^{3}$. The details of different load demands including power, heat, and gas are shown in Figure 2. The forecasted wind power and the price of the grid are shown in Figure 3 . The maximum allowable fluctuation is set to $10 \%$.

4.1. Experimental Results of Robust Optimization Regarding Wind Power Utilization. In this section, to demonstrate the effectiveness of the presented approach for reducing wind power curtailment, the optimized results of the outputs of SS, CG, and the exchange power of the two-stage robust model are shown in Figures 4 and 5. Figure 4 indicates that when the wind power is plentiful during the $1^{\text {st }}-7^{\text {th }}$ and the $22^{\text {nd }}-24^{\text {th }}$ hours, the excessive wind power is converted into heat and gas energy by EB and PtG, respectively. Therefore, the wind power utilization is considerably promoted. Figure 5 shows that ESS successfully improves the wind power 
TABLE 3: The solution of the model using C\&CG.

Step 1 Initialize lower and upper bounds $\mathrm{UB}=+\infty$ and $\mathrm{LB}=-\infty$. Set the iteration no. $k=0$ and the convergence gap $\varepsilon>0$.

Step 2 Solve MP. Derive an optimal solution $\left(\mathbf{x}_{k}^{*}, \eta_{k}, \mathbf{y}_{1}, \ldots, \mathbf{y}_{k}\right)$, and update the lower bound $L B=\mathbf{a}^{\mathrm{T}} \mathbf{x}_{k}+\eta_{k}$.

Step 3 Solve SP3 with respect to the fixed $\left(\mathbf{x}_{k}^{*}, \eta, \mathbf{y}_{1}, \ldots, \mathbf{y}_{k}\right)$ from MP. Obtain the solution $\left(\mathbf{u}_{k}^{o}, \mathbf{y}_{k}^{o}\right)$ and update $\mathbf{u}_{k+1}=\mathbf{u}_{k}^{o}$ and UB $=$ min $\left\{\mathbf{c}^{\mathrm{T}} \mathbf{x}_{k}^{*}+\mathbf{a}^{\mathrm{T}} \mathbf{y}_{k}^{o}+\mathbf{b}^{\mathrm{T}} \mathbf{u}_{k}^{o}, \mathrm{UB}\right\}$.

Step 4 If $\mathrm{UB}-\mathrm{LB} \leq \varepsilon$, return the solution $\mathbf{x}=\mathbf{x}_{k}^{*}$ and the algorithm terminates. Otherwise, create a variable $\mathbf{y}_{k+1}$ and add constraint equations (27)-(29) to MP. And then update $k=k+1$ and go back to step 2.

TABLE 4: The parameters of CGs

\begin{tabular}{lccccccc}
\hline CG & $P^{\max }(\mathrm{kW} / \mathrm{h})$ & $P^{\min }(\mathrm{kW} / \mathrm{h})$ & $R^{\text {up }}(\mathrm{kW} / \mathrm{h})$ & $R^{\text {down }}(\mathrm{kW} / \mathrm{h})$ & $a$ & $b$ & $\lambda_{\mathrm{CG}}(\$ / \mathrm{MWh})$ \\
\hline Microturbine & 600 & 0 & 360 & 360 & - & - & 1.35 \\
FC & 400 & 0 & 120 & 120 & 0.002 & 0.85 \\
EB & 280 & 0 & 200 & 200 & - & - & 1.15 \\
PtG & 100 & 0 & 100 & 100 & - & - \\
\hline
\end{tabular}

TABLE 5: The parameters of SS.

\begin{tabular}{lccccc}
\hline SS & $P_{\mathrm{SS}}^{\max }(\mathrm{kW} / \mathrm{h})$ & $E_{\mathrm{SS}}^{\max }(\mathrm{kWh})$ & $E_{\mathrm{SS}}^{\min }(\mathrm{kWh})$ & $E_{\text {initial }}(\mathrm{kWh})$ & 320 \\
\hline ESS & 600 & 1600 & 20 & $\eta_{\mathrm{ch} / \mathrm{dis}}(\$ / \mathrm{kWh})$ \\
TSS & 400 & 1000 & 20 & 360 & $0.95 / 1.15$ \\
\hline
\end{tabular}

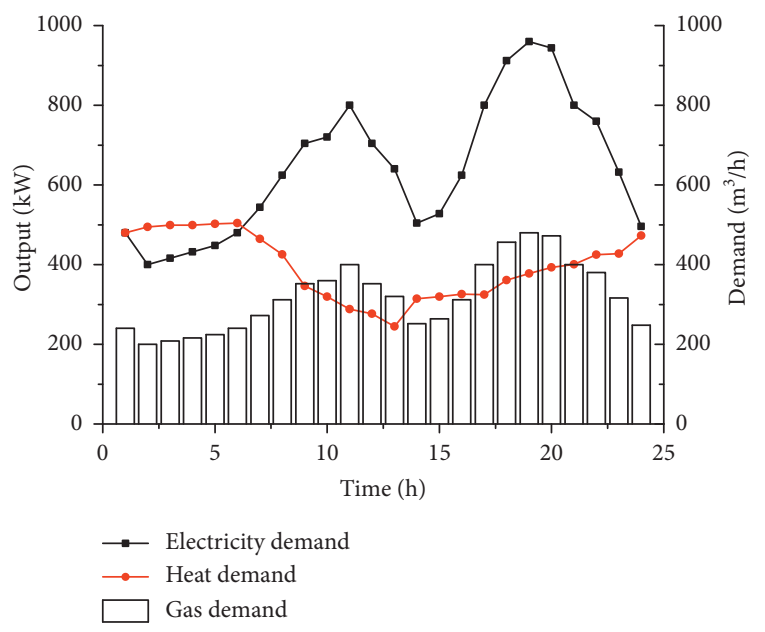

Figure 2: Load demands.

accommodation when the wind power is plentiful during the $1^{\text {st }}-6^{\text {th }}$ and the $21^{\text {st }}-23^{\text {rd }}$ hours. The reason is that during these periods, ESS is able to keep charging to store the unconsumed wind power. Moreover, HSS keeps discharging heat power in the $23^{\text {rd }}-24^{\text {th }}$ hours. Consequently, the electricity and the heat outputs of the microturbine decrease at the same time, which creates an extra margin for wind power accommodation.

Figure 5 indicates that MG sells power to the grid when the wind is plentiful, which improves the wind power utilization. Additionally, MG also sells power when the sold price is relatively high during the $9^{\text {th }}-11^{\text {th }}$ hours and $19^{\text {th }}-23^{\text {rd }}$ hours. Therefore, the profit of MG can be significantly improved.

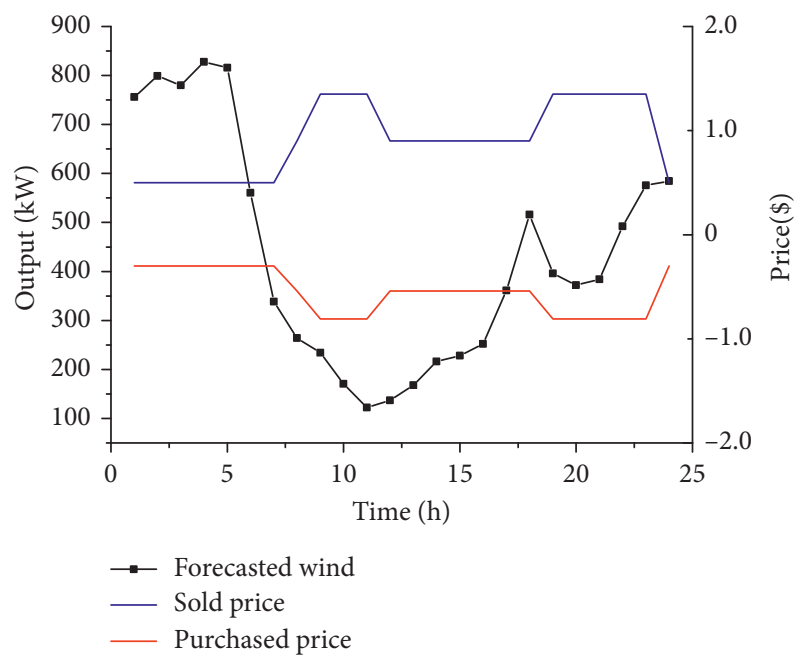

Figure 3: Forecasted wind power and the price of grid.

4.2. Impact of Uncertain Budget. In this section, different values of the uncertain budget $\Gamma=0,8,16$, and 24 are employed to study its impact on optimal day-ahead ED. In our model, $\Gamma$ represents the allowable fluctuation times of the wind power. For example, if $\Gamma$ equals 0 , it indicates that in the period $T$, wind power should equal its forecasted wind power. If $\Gamma$ equals 8 , it means that the fluctuation times of the wind power should be no more than 8 times. As long as the optimal day-ahead ED strategy is achieved, another 500 scenarios are generated by Monte Carlo simulation as testing scenarios to evaluate the performance of the achieved optimal strategy. The optimized results based on different values of $\Gamma$ are shown in Table 6 . 


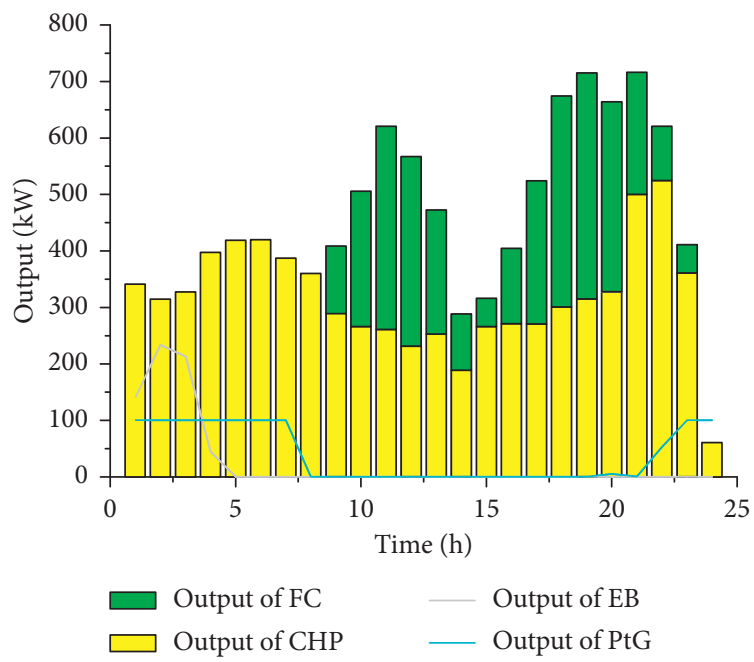

Figure 4: Outputs of CG of the robust model.

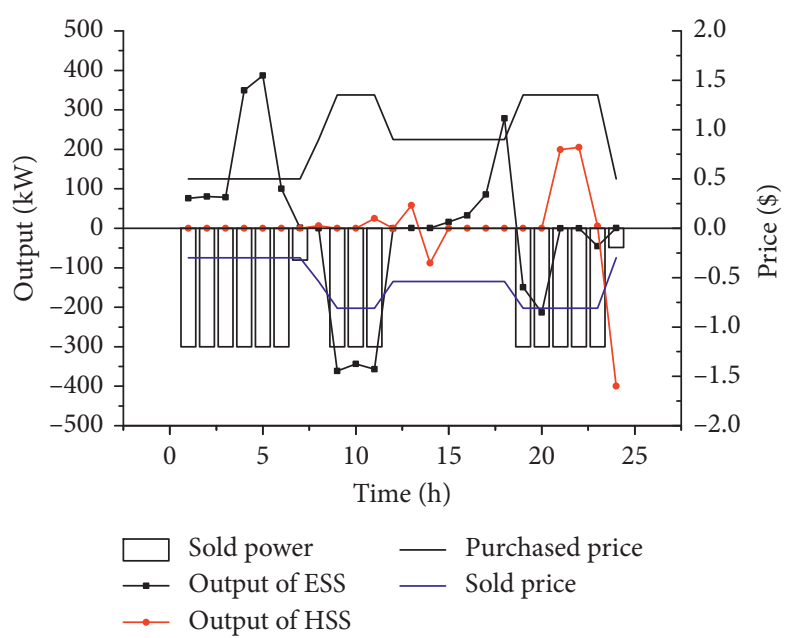

FIGURE 5: Outputs of SS of the robust model.

TABle 6: Optimization results under different uncertain budget.

\begin{tabular}{|c|c|c|c|c|c|c|c|c|c|}
\hline \multirow{2}{*}{\multicolumn{2}{|c|}{$\begin{array}{l}\text { Uncertain } \\
\text { budget }\end{array}$}} & \multicolumn{2}{|c|}{$\Gamma=0$} & \multicolumn{2}{|c|}{$\Gamma=8$} & \multicolumn{2}{|c|}{$\Gamma=16$} & \multicolumn{2}{|c|}{$\Gamma=24$} \\
\hline & & Mean & $\operatorname{Max}$ & Mean & $\operatorname{Max}$ & Mean & $\operatorname{Max}$ & Mean & Max \\
\hline \multicolumn{2}{|c|}{ Day-ahead operation cost $(\$)$} & \multicolumn{2}{|c|}{13165.8} & \multicolumn{2}{|c|}{13196.2} & \multicolumn{2}{|c|}{13233.1} & \multicolumn{2}{|c|}{13260.2} \\
\hline \multirow{4}{*}{$\begin{array}{l}\text { Day-ahead } \\
\text { operation cost }(\$)\end{array}$} & Cost of gas & \multicolumn{2}{|c|}{13343.3} & \multicolumn{2}{|c|}{13255.3} & \multicolumn{2}{|c|}{13149.6} & \multicolumn{2}{|c|}{13071.6} \\
\hline & Cost of FC & \multicolumn{2}{|c|}{2345.1} & \multicolumn{2}{|c|}{2463.5} & \multicolumn{2}{|c|}{2606.2} & \multicolumn{2}{|c|}{2711.3} \\
\hline & Cost of grid & \multicolumn{2}{|c|}{$-2.522 .7$} & \multicolumn{2}{|c|}{-2522.7} & \multicolumn{2}{|c|}{-2522.7} & \multicolumn{2}{|c|}{-2522.7} \\
\hline & $\begin{array}{l}\text { Cost of wind } \\
\text { curtailment }\end{array}$ & \multicolumn{2}{|c|}{0} & \multicolumn{2}{|c|}{0} & \multicolumn{2}{|c|}{0} & \multicolumn{2}{|c|}{0} \\
\hline \multicolumn{2}{|c|}{ Adjustment cost $(\$)$} & 241.6 & 473.4 & 158.9 & 322.0 & 129.7 & 360.2 & 121.1 & 313.5 \\
\hline \multicolumn{2}{|c|}{ Total cost $(\$)$} & 13407.4 & 13639.2 & 13355.1 & 13518.2 & 13362.8 & 13593.3 & 13381.1 & 13573.7 \\
\hline
\end{tabular}

Table 6 demonstrates that the day-ahead operation cost of the robust optimization keeps increasing along with the uncertain budget value rises from 0 to 24 . As $\Gamma$ is highly related to the number of the fluctuations, an increasing $\Gamma$ leads to more fluctuation times of the wind power, which means wind power is more likely to become
TABLE 7: Scheduled output of two-stage robust model under different uncertain budgets.

\begin{tabular}{lcccc}
\hline Model (kW) & $\Gamma=0$ & $\Gamma=8$ & $\Gamma=16$ & $\Gamma=24$ \\
\hline Scheduled microturbine output & 7650.6 & 7651.1 & 7651.7 & 7651.6 \\
Scheduled FC output & 3126.9 & 3264.8 & 3474.9 & 3615.1 \\
\hline
\end{tabular}


TABLE 8: Optimization results with different forecasted error.

\begin{tabular}{|c|c|c|c|c|c|c|c|c|c|}
\hline \multirow{2}{*}{\multicolumn{2}{|c|}{ Forecasted error }} & \multicolumn{2}{|c|}{ Error $=5 \%$} & \multicolumn{2}{|c|}{ Error $=10 \%$} & \multicolumn{2}{|c|}{ Error $=15 \%$} & \multicolumn{2}{|c|}{ Error $=20 \%$} \\
\hline & & Mean & Max & Mean & $\operatorname{Max}$ & Mean & Max & Mean & Max \\
\hline \multicolumn{2}{|c|}{ Day-ahead operation cost (\$) } & \multicolumn{2}{|c|}{13180.9} & \multicolumn{2}{|c|}{13196.2} & \multicolumn{2}{|c|}{13211.2} & \multicolumn{2}{|c|}{13226.2} \\
\hline \multirow{4}{*}{ Day-ahead operation cost (\$) } & Cost of gas & \multicolumn{2}{|c|}{13298.8} & \multicolumn{2}{|c|}{13255.3} & \multicolumn{2}{|c|}{13211.2} & \multicolumn{2}{|c|}{13166.0} \\
\hline & Cost of FC & \multicolumn{2}{|c|}{2404.8} & \multicolumn{2}{|c|}{2463.5} & \multicolumn{2}{|c|}{2522.6} & \multicolumn{2}{|c|}{2582.9} \\
\hline & Cost of grid & \multicolumn{2}{|c|}{-2522.7} & \multicolumn{2}{|c|}{-2522.7} & \multicolumn{2}{|c|}{-2522.7} & \multicolumn{2}{|c|}{-2522.7} \\
\hline & Cost of wind curtailment & \multicolumn{2}{|c|}{0} & \multicolumn{2}{|c|}{0} & \multicolumn{2}{|c|}{0} & \multicolumn{2}{|c|}{0} \\
\hline \multicolumn{2}{|c|}{ Adjustment cost $(\$)$} & 81.5 & 189.8 & 158.9 & 322.0 & 271.7 & 630.5 & 358.4 & 648.7 \\
\hline \multicolumn{2}{|c|}{ Total cost $(\$)$} & 13262.4 & 13370.7 & 13355.1 & 13518.2 & 13482.9 & 13841.7 & 13584.6 & 13874.9 \\
\hline
\end{tabular}

TABLE 9: Optimization results with different confidence intervals.

\begin{tabular}{|c|c|c|c|c|c|c|c|c|c|}
\hline \multirow{2}{*}{\multicolumn{2}{|c|}{ Confidence interval }} & \multicolumn{2}{|c|}{$80 \%$} & \multicolumn{2}{|c|}{$85 \%$} & \multicolumn{2}{|c|}{$90 \%$} & \multicolumn{2}{|c|}{$95 \%$} \\
\hline & & Mean & Max & Mean & $\operatorname{Max}$ & Mean & $\operatorname{Max}$ & Mean & $\operatorname{Max}$ \\
\hline \multicolumn{2}{|c|}{ Day-ahead operation cost $(\$)$} & \multicolumn{2}{|c|}{13213.9} & \multicolumn{2}{|c|}{13200.2} & \multicolumn{2}{|c|}{13194.3} & \multicolumn{2}{|c|}{13187.2} \\
\hline \multirow{4}{*}{ Day-ahead operation cost $(\$)$} & Cost of gas & \multicolumn{2}{|c|}{13176.4} & \multicolumn{2}{|c|}{13168.3} & \multicolumn{2}{|c|}{13274} & \multicolumn{2}{|c|}{13295} \\
\hline & Cost of FC & \multirow{2}{*}{\multicolumn{2}{|c|}{$\begin{array}{c}2560.2 \\
-2522.7\end{array}$}} & \multicolumn{2}{|c|}{2554.6} & \multicolumn{2}{|c|}{2443.0} & \multicolumn{2}{|c|}{2414.9} \\
\hline & Cost of grid & & & -25 & 22.7 & -25 & 2.7 & -252 & 2.7 \\
\hline & Cost of wind curtailment & \multicolumn{2}{|c|}{0} & \multicolumn{2}{|c|}{0} & \multicolumn{2}{|c|}{0} & \multicolumn{2}{|c|}{0} \\
\hline \multicolumn{2}{|c|}{ Adjustment cost (\$) } & 295.4 & 591.2 & 222.4 & 535.2 & 138.4 & 262.3 & 98.2 & 230.2 \\
\hline \multicolumn{2}{|c|}{ Total cost $(\$)$} & 13509.3 & 13805.1 & 13422.6 & 13735.4 & 13332.7 & 13456.6 & 13285.4 & 13417.4 \\
\hline
\end{tabular}

insufficient in the period $T$. Therefore, as shown in Table 7 , the total scheduled output of generators becomes higher to immunize against the wind power uncertainty. Although the increasing values of $\Gamma$ guarantee the robustness of the model, the day-ahead operation cost becomes higher due to the increase in the scheduled output of the microturbine and FC. To sum up, larger $\Gamma$ leads to worse economy but better robustness of the ED strategy. Therefore, the uncertainty budget can be flexibly adjusted to balance the economy and robustness of the ED strategy in practical applications.

4.3. Impact of the Forecasted Error. In this section, different maximum forecasted errors $5 \%, 10 \%, 15 \%$, and $20 \%$ of the wind power are considered to reveal its impact on the dayahead ED. Also 500 scenarios are generated as testing scenarios to evaluate the economy of the achieved optimal strategy. The optimized results based on different forecasted errors are illustrated in Table 8.

As shown in Table 8, the day-ahead operation cost and the adjustment cost increase along with the increasing forecasted error. This is due to the fact that with the increasing forecasted error, the worst-case wind power becomes more insufficient. As a result, more resources and adjustment output are scheduled to handle the uncertain wind power in the robust optimization model, which causes the increasing costs.

4.4. Impact of the Confidence Interval. In this section, confidence intervals $80 \%, 85 \%, 90 \%$, and $95 \%$ of the wind power are considered to reveal its impact on the day-ahead ED. 500 scenarios are generated as testing scenarios to evaluate the effectiveness of the achieved optimal strategy. The optimized results based on different confidence intervals are illustrated in Table 9.

As shown in Table 9, the day-ahead operation cost and the adjustment cost decrease steadily as the confidence interval increases. This reveals the fact that with the increasing confidence interval, the uncertainty set narrows and the value of the worst-case wind power is closer to the forecasted wind power. Therefore, fewer resources and adjustment output are needed to handle the uncertain wind power, which reduces the total operation cost.

4.5. Optimization Comparison with Different Approaches. In this section, the deterministic model and the single-stage robust model are also implemented in terms of comparison. Their cost reduction optimization performances are compared to those of the presented two-stage robust model. Both the uncertainty budgets of the single-stage and the two-stage robust model are set to 8 . The comparisons of different approaches are shown in Table 10.

According to Table 10, the cost of the two-stage robust model is lower than that of the single-stage model. The single-stage model schedules less wind power and more generation output than those of the two-stage robust model, as shown in Table 11. This is due to the fact that the singlestage model only considers one worst-case wind power scenario. However, the two-stage robust model is able to search for a branch of worst-case wind power scenarios by iterations between the first stage and the second stage. Therefore, the two-stage model is less conservative than the single-stage model. In other words, the two-stage robust 
TABle 10: Optimization results of different approaches.

\begin{tabular}{|c|c|c|c|c|c|c|c|}
\hline \multirow{2}{*}{\multicolumn{2}{|c|}{ Model }} & \multicolumn{2}{|c|}{ Two-stage robust } & \multicolumn{2}{|c|}{ Single-stage robust } & \multicolumn{2}{|c|}{ Deterministic } \\
\hline & & Mean & Max & Mean & Max & Mean & Max \\
\hline \multicolumn{2}{|c|}{ Day-ahead operation cost $(\$)$} & \multicolumn{2}{|c|}{13196.2} & \multicolumn{2}{|c|}{13384.2} & \multicolumn{2}{|c|}{13165.8} \\
\hline \multirow{4}{*}{ Day-ahead operation cost $(\$)$} & Cost of gas & \multicolumn{2}{|c|}{13255.3} & \multicolumn{2}{|c|}{13460.3} & \multicolumn{2}{|c|}{13165.8} \\
\hline & Cost of FC & \multicolumn{2}{|c|}{2463.5} & \multicolumn{2}{|c|}{2427.1} & \multicolumn{2}{|c|}{2345.1} \\
\hline & Cost of grid & \multicolumn{2}{|c|}{-2522.7} & \multicolumn{2}{|c|}{-2503.2} & \multicolumn{2}{|c|}{$-2.522 .7$} \\
\hline & Cost of wind curtailment & \multicolumn{2}{|c|}{0} & \multicolumn{2}{|c|}{0} & \multicolumn{2}{|c|}{0} \\
\hline \multicolumn{2}{|c|}{ Adjustment cost $(\$)$} & 158.9 & 322.0 & 308.2 & 497.7 & 241.6 & 473.4 \\
\hline \multicolumn{2}{|c|}{ Total cost $(\$)$} & 13355.1 & 13518.2 & 13692.4 & 13881.9 & 13407.4 & 13639.2 \\
\hline
\end{tabular}

TABLE 11: Results of the single-stage and two-stage robust models.

\begin{tabular}{lcc}
\hline Model $(\mathrm{kW})$ & $\begin{array}{c}\text { Single-stage } \\
\text { robust }\end{array}$ & $\begin{array}{c}\text { Two-stage } \\
\text { robust }\end{array}$ \\
\hline Scheduled microturbine & 7767.6 & 7651.1 \\
output & 3236.2 & 3264.8 \\
Scheduled FC output & 9785.6 & 10351.1 \\
\hline
\end{tabular}

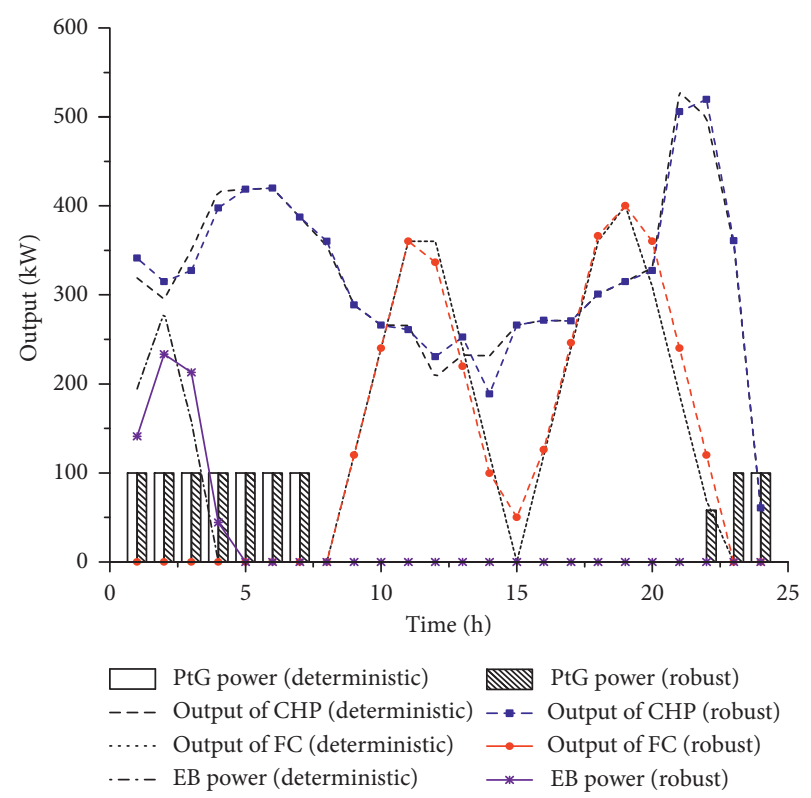

FIGURE 6: Outputs of CG of the robust model and the deterministic model.

model can better balance the robustness and economy than those of the single-stage model.

It is also can be observed that the day-ahead operation cost of the two-stage robust model is higher than that of the deterministic model. The two-stage robust model considers the worst scenarios of the uncertain wind power. Therefore, more resources and more operation cost are needed in the day-ahead strategy, but less adjustment cost is demanded, as shown in Figure 6. In this way, the total operation cost of the two-stage robust model is effectively reduced compared to that of the deterministic model.

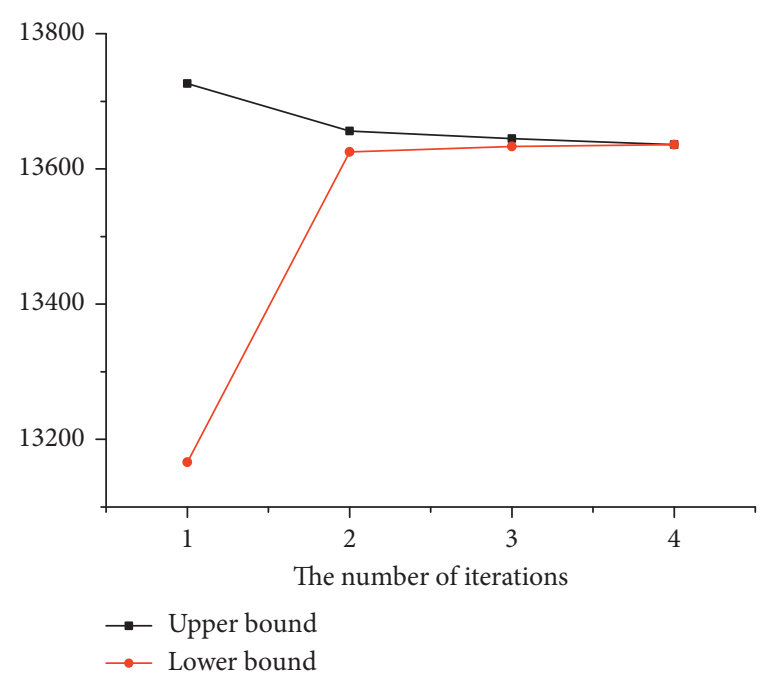

FIGURE 7: Iterations of the two-stage robust model solution.

Figure 7 shows that during the two-stage robust model solution, the presented C\&CG algorithm converges to the global optimal solution after only 4 iterations. This point significantly indicates the efficiency of the presented approach.

\section{Conclusion}

This paper presents a two-stage robust model in enabling optimal day-ahead ED for integrated power-heat-gas MG considering wind power. To be aware of the natural characteristics of wind, this paper employs an uncertainty set to describe the uncertainty of wind power. Additionally, to facilitate the problem solution, the original model with a complex min-max-min structure is decomposed into MP and SP with min and max-min structures, respectively, by the C\&CG algorithm. Based on the duality theory and Big-M approach, the original SP can be successfully linearized. Therefore, the decomposed model can be directly solved by iterations between SP and MP. Experimental results demonstrate that the two-stage robust model can handle the worst-case wind power scenarios to achieve the optimal dayahead ED strategy. Compared to the single-stage robust model and the deterministic model, the presented approach can ensure a less-conservative and more-convincible solution. Moreover, the budget of uncertainty enables the 
adjustment of the optimization performance for the dispatch scheme, so that the robustness and the economy of the ED strategy can be better balanced. In our future work, cooling energy supplying and uncertainty of load demands will be further explored.

\section{Data Availability}

The data of the models and algorithms used to support the findings of this study are included within the article.

\section{Conflicts of Interest}

The authors declare that there are no conflicts of interest regarding the publication of this article.

\section{References}

[1] C. Peng, Z. Zhang, and J. Wu, "A wind power and load prediction based frequency control approach for wind-dieselbattery hybrid power system," Mathematical Problems in Engineering, vol. 2015, no. 7, Article ID 715435, 2015.

[2] Y. Yang, "Practical robust optimization method for unit commitment of a system with integrated wind resource," Mathematical Problems in Engineering, vol. 2017, Article ID 5208290, 13 pages, 2017.

[3] R. H. Lasseter, "IEEE power engineering society winter meeting," in Proceedings of the 2002 IEEE Power Engineering Society Winter Meeting Conference Proceedings (Cat No 02CH37309) PESW-02, vol. 1, pp. 305-308, New York, NY, USA, January 2002.

[4] M. Liserre, T. Sauter, and J. Hung, "Future energy systems: integrating renewable energy sources into the smart power grid through industrial electronics," IEEE Industrial Electronics Magazine, vol. 4, no. 1, pp. 18-37, 2010.

[5] H. Li, A. T. Eseye, J. Zhang, and D. Zheng, "Optimal energy management for industrial microgrids with high-penetration renewables," Protection and Control of Modern Power Systems, vol. 2, no. 2, pp. 122-135, 2017.

[6] Z. Li, W. Wu, M. Shahidehpour, J. Wang, and B. Zhang, "Combined heat and power dispatch considering pipeline energy storage of district heating network," IEEE Transactions on Sustainable Energy, vol. 7, no. 1, pp. 12-22, 2016.

[7] J. Aghaei and M.-I. Alizadeh, "Multi-objective self-scheduling of CHP (combined heat and power)-based microgrids considering demand response programs and ESSs (energy storage systems)," Energy, vol. 55, no. 15, pp. 1044-1054, 2013.

[8] H. R. Abdolmohammadi and A. Kazemi, "A benders decomposition approach for a combined heat and power economic dispatch," Energy Conversion and Management, vol. 71, pp. 21-31, 2013.

[9] M. G. Nielsen, J. M. Morales, M. Zugno, T. E. Pedersen, and H. Madsen, "Economic valuation of heat pumps and electric boilers in the Danish energy system," Applied Energy, vol. 167, no. 1, pp. 189-200, 2016.

[10] C. He, L. Wu, T. Liu, and M. Shahidehpour, "Robust cooptimization scheduling of electricity and natural gas systems via ADMM," IEEE Transactions on Sustainable Energy, vol. 8, no. 2, pp. 658-670, 2017.

[11] C. He, L. Wu, T. Liu, W. Wei, and C. Wang, "Co-optimization scheduling of interdependent power and gas systems with electricity and gas uncertainties," Energy, vol. 159, no. 15, pp. 1003-1015, 2018.
[12] J. Ye and R. Yuan, "Integrated natural gas, heat, and power dispatch considering wind power and power-to-gas," Sustainability, vol. 9, no. 4, p. 602, 2017.

[13] Y. Li, Y. Zou, Y. Tan et al., "Optimal stochastic operation of integrated low-carbon electric power, natural gas, and heat delivery system," IEEE Transactions on Sustainable Energy, vol. 9, no. 1, pp. 273-283, 2018.

[14] J. He, Y. Liu, and Y. Liu, "A position-level global optimization inverse kinematic solution algorithm for dual redundant robots based on motion characteristics," Mathematical Problems in Engineering, vol. 2018, Article ID 3854812, 10 pages, 2018.

[15] G. Li, R. Zhang, T. Jiang et al., "Optimal dispatch strategy for integrated energy systems with CCHP and wind power," Applied Energy, vol. 192, pp. 408-419, 2017.

[16] W. Peng and Y. Liu, "The integration of wind-solar-hydropower generation in enabling economic robust dispatch," Mathematical Problems in Engineering, vol. 2019, Article ID 4634131, 12 pages, 2019.

[17] L. Feng, J. Zhang, G. Li et al., "Cost reduction of a hybrid energy storage system considering correlation between wind and PV power," Protection and Control of Modern Power Systems, vol. 1, no. 1, pp. 86-94, 2016.

[18] J. Li, S. Wang, L. Ye, and J. Fang, "A coordinated dispatch method with pumped-storage and battery-storage for compensating the variation of wind power," Protection and Control of Modern Power Systems, vol. 3, no. 3, pp. 21-34, 2018.

[19] Y. Chen, W. Wei, F. Liu, and S. Mei, "Distributionally robust hydro-thermal-wind economic dispatch," Applied Energy, vol. 173, no. 1, pp. 511-519, 2016.

[20] Y. J. Zhang, Y. Y. Yang, and Z. H. Liu, “A joint day-ahead scheduling for photovoltaic-storage systems based on extended QV bus-type power flow," in Proceedings of the 2015 International Conference on Smart Grid and Clean Energy Technologies (ICSGCE), pp. 18-22, Offenburg, Germany, October 2015.

[21] G. Li, R. Zhang, T. Jiang, H. Chen, L. Bai, and X. Li, "Securityconstrained bi-level economic dispatch model for integrated natural gas and electricity systems considering wind power and power-to-gas process," Applied Energy, vol. 194, pp. 696-704, 2017.

[22] B. Li, X. Qian, J. Sun, K. L. Teo, and C. Yu, "A model of distributionally robust two-stage stochastic convex programming with linear recourse," Applied Mathematical Modelling, vol. 58, pp. 86-97, 2018.

[23] D. Bertsimas, M. Sim, and M. Zhang, "Adaptive distributionally robust optimization," Management Science, vol. 65, no. 2, pp. 604-618, 2018.

[24] A. Ben-Tal, L. El. Ghaoui, and A. Nemirovski, Robust Optimization, Princeton University Press, Princeton, NJ, USA, 2009.

[25] A. K. Basu, A. Bhattacharya, S. Chowdhury, and S. P. Chowdhury, "Planned scheduling for economic power sharing in a CHP-based micro-grid," IEEE Transactions on Power Systems, vol. 27, no. 1, pp. 30-38, 2012.

[26] A. Haghrah, M. Nazari-Heris, and B. Mohammadi-Ivatloo, "Solving combined heat and power economic dispatch problem using real coded genetic algorithm with improved Mühlenbein mutation," Applied Thermal Engineering, vol. 99, pp. 465-475, 2016.

[27] W. J. Shen and H. X. Li, "Multi-scale parameter identification of lithium-ion battery electric models using a pso-lm algorithm," Energies, vol. 10, no. 4, p. 432, 2017. 
[28] A. Bayat and A. Bagheri, "Optimal active and reactive power allocation in distribution networks using a novel heuristic approach," Applied Energy, vol. 233-234, pp. 71-85, 2019.

[29] C. Peng, P. Xie, J. Zhan et al., "Robust economic dispatch of microgrid using improved bacterial foraging algorithm," Power System Technology, vol. 38, no. 9, pp. 2392-2398, 2014.

[30] D. Sun, L. Zhang, D. Su et al., "Two-stage robust securityconstrained unit commitment with optimizable interval of uncertain wind power output," Mathematical Problems in Engineering, vol. 2017, Article ID 9153297, 11 pages, 2017.

[31] F. Liu, Z. Bie, and X. Wang, "Day-ahead dispatch of integrated electricity and natural gas system considering reserve scheduling and renewable uncertainties," IEEE Transactions on Sustainable Energy, vol. 10, no. 2, pp. 646-658, 2019.

[32] Y. Zhang, N. Gatsis, and G. B. Giannakis, "Robust energy management for microgrids with high-penetration renewables," IEEE Transactions on Sustainable Energy, vol. 4, no. 4, pp. 944-953, 2013.

[33] W. Wei, F. Liu, and S. Mei, "Distributionally robust co-optimization of energy and reserve dispatch," IEEE Transactions on Sustainable Energy, vol. 7, no. 1, pp. 289-300, 2016.

[34] S. Campanari and E. Macchi, "Technical and tariff scenarios effect on microturbine trigenerative applications," Journal of Engineering for Gas Turbines and Power, vol. 126, no. 3, pp. 581-589, 2004.

[35] Y. Shui, H. Gao, L. Wang, Z. Wei, and J. Liu, "A data-driven distributionally robust coordinated dispatch model for integrated power and heating systems considering wind power uncertainties," International Journal of Electrical Power \& Energy Systems, vol. 104, pp. 255-258, 2019.

[36] Y. Li, Y. Zou, and Y. Tan, "Optimal stochastic operation of integrated low-carbon electric power, natural gas, and heat delivery system," IEEE Transactions on Sustainable Energy, vol. 9, no. 1, pp. 273-283, 2017.

[37] X. Xiao, Y. Shui, H. Gao et al., "A two-stage distributionally robust coordinated dispatch for integrated electricity and natural-gas energy systems considering uncertainty of wind power," IOP Conference Series: Materials Science and Engineering, vol. 366, no. 1, pp. 12-16, 2018.

[38] B. Zhang, Q. Li, L. Wang, and W. Feng, "Robust optimization for energy transactions in multi-microgrids under uncertainty," Applied Energy, vol. 217, pp. 346-360, 2018. 